\title{
Sinus floor elevation utilizing the transalveolar approach
}

\author{
Pjetursson, Bjarni E ; Lang, Niklaus P
}

\begin{abstract}
A transalveolar approach for sinus floor elevation with subsequent placement of dental implants was first suggested by Tatum in 1986. In 1994, Summers described a different transalveolar approach using a set of tapered osteotomes with increasing diameters. The transalveolar approach of sinus floor elevation, also referred to as 'osteotome sinus floor elevation', the 'Summers technique' or the 'Crestal approach', may be considered as being more conservative and less invasive than the conventional lateral window approach. This is reflected by the fact that more than nine out of 10 patients who experienced the surgical procedure would be willing to undergo it again. The main indication for transalveolar sinus floor elevation is reduced residual bone height, which does not allow standard implant placement. Contraindications for transalveolar sinus floor elevation may be intra-oral, local or medical. The surgical approach utilized over the last two decades is the technique described by Summers, with or without minor modifications. The surgical care after implant placement using the osteotome technique is similar to the surgical care after standard implant placement. The patients are usually advised to take antibiotic prophylaxis and to utilize antiseptic rinses. The main complications reported after performing a transalveolar sinus floor elevation were perforation of the Schneiderian membrane in $3.8 \%$ of patients and postoperative infections in $0.8 \%$ of patients. Other complications reported were postoperative hemorrhage, nasal bleeding, blocked nose, hematomas and benign paroxysmal positional vertigo. Whether it is necessary to use grafting material to maintain space for new bone formation after elevating the sinus membrane utilizing the osteotome technique is still controversial. Positive outcomes have been reported with and without using grafting material. A prospective study, evaluating both approaches, concluded that significantly more bone gain was seen when grafting material was used ( $4.1 \mathrm{~mm}$ mean bone gain compared with $1.7 \mathrm{~mm}$ when no grafting material was utilized). In a systematic review, including 19 studies reporting on 4388 implants inserted using the transalveolar sinus floor elevation technique, the 3-year implant survival rate was 92.8\% (95\% confidence interval: 87.4-96.0\%). Furthermore, a subject-based analysis of the same material revealed an annual failure rate of $3.7 \%$. Hence, one in 10 subjects experienced implant loss over 3 years. Several of the included studies demonstrated that transalveolar sinus floor elevation was most predictable when the residual alveolar bone height was $\geq 5 \mathrm{~mm}$ and the sinus floor anatomy was relatively flat.
\end{abstract}

DOI: https://doi.org/10.1111/prd.12043

Posted at the Zurich Open Repository and Archive, University of Zurich

ZORA URL: https://doi.org/10.5167/uzh-107994

Journal Article

Accepted Version

Originally published at:

Pjetursson, Bjarni E; Lang, Niklaus P (2014). Sinus floor elevation utilizing the transalveolar approach. Periodontology 2000, 66(1):59-71.

DOI: https://doi.org/10.1111/prd.12043 


\title{
Sinus floor elevation utilizing the trans-alveolar approach
}

\author{
Bjarni E. Pjetursson ${ }^{1}$, \& Niklaus P. Lang ${ }^{2}$
}

${ }^{1}$ University of Iceland, Faculty of Odontology, Reykjavik, Iceland

${ }^{2}$ The University of Hong Kong, Faculty of Dentistry, Hong Kong SAR PR China

Corresponding address:

Bjarni E. Pjetursson, DDS, Dr.med.dent., MAS (Perio)

Department of Reconstructive Dentistry

University of Iceland

Vatnsmyrarvegur 16

IS-101 Reykjavik, Iceland

bep@hi.is

Phone: +354 5254850

Fax: +3545254870 


\section{Authors}

Prof. Bjarni E. Pjetursson, DDS, Dr.med.dent., MAS

Head of the Department of Reconstructive Dentistry

Vice Dean

University of Iceland

Reykjavik, Iceland

Prof. Niklaus P. Lang, DMD, MS, PhD, Dr. odont. h.c.

Program director of implant dentistry

University of Hong Kong

Prince Phillip Dental Hospital

Hong Kong, SAR PRC China 


\begin{abstract}
A trans-alveolar approach for sinus floor elevation with subsequent placement of dental implants was first suggested by Tatum in 1986. Later on, Summers (1994) described a different transalveolar approach using set of tapered osteotomes with increasing diameters. The trans-alveolar approach, also referred to as "Osteotome Sinus Floor Elevation (OSFE)", the "Summers technique" or "Crestal approach" may be considered more conservative and less invasive than the conventional lateral window approach of sinus floor elevation. This is reflected in the fact that more then 9 out of 10 patients that have experienced the surgical procedure were willing to undergo it again.

The main indication for trans-alveolar sinus floor elevation is reduced residual bone height, which does not allow standard implant placement. Contraindications for trans-alveolar sinus floor elevation may by intraoral contraindications, local contraindications or medical conditions.

The surgical approach utilized over the last two decades is the technique described by Summers with or without minor modifications. The post-surgical care after implant placement with the osteotome technique is similar to the post-surgical care after standard implant placement. The patients are usually advised to take antibiotic prophylaxis and utilize antiseptic rinses. The main complications reported after performing a trans-alveolar sinus floor elevation are perforation of the Schneiderian membrane, occurring in $3.8 \%$ of the cases and post-operative infections occurring in $0.8 \%$ of the cases. Other complications reported were post-operative haemorrhage, nasal bleeding, blocked nose, hematomas and benign paroxysmal positional vertigo.

It is still controversial, whether it is necessary to use grafting material to maintain the space for new bone formation after elevating the sinus membrane utilizing the osteotome technique. Positive outcomes have been reported with and without using grafting material.
\end{abstract}

A prospective study evaluating both approaches concluded that significantly more bone gain was seen when grafting material was used $(4.1 \mathrm{~mm}$ mean bone gain compared to $1.7 \mathrm{~mm}$ when no grafting material was utilized).

In a systematic review, including 19 studies reporting on 4388 implants inserted using the transalveolar sinus floor elevation technique, the 3 -year implant survival rate was $92.8 \%$ (95\% C.I.: $87.4 \%-96.0 \%$ ). Furthermore, a subject-based analysis of the same material revealed an annual failure rate of $3.7 \%$. Hence, one out of ten subjects experienced implant loss over 3 years.

Several of the included studies demonstrated that trans-alveolar sinus floor elevation was most predictable when the residual alveolar bone height was $5 \mathrm{~mm}$ or more and the sinus floor anatomy was relatively flat. 


\section{Introduction}

Elevation of the maxillary sinus floor was first reported by Boyne in the 1960s. Fifteen years later, Boyne and James (1980) described the elevation of the maxillary sinus floor in patients with large, pneumatized sinus cavities as a preparation for the placement of blade implants. The authors described a two-stage procedure, where the maxillary sinus was grafted using autogenous particulate iliac bone at the first stage of surgery. In the second stage of surgery, after approximately 3 months, the blade implants were placed and later used to support fixed or removable reconstructions (3).

As implant dentistry developed, it became more evident that the posterior maxillary region was often limited for standard implant placement, since the residual vertical bone height was substantially reduce. An elevation of the maxillary sinus floor was in the treatment for solving this problem. Several surgical techniques have been presented for entering the sinus cavity, elevating the sinus membrane, and placing dental implants with or without the application of bone grafts.

A trans-alveolar approach for sinus floor elevation, with subsequent placement of implants, was first suggested by Tatum in 1986 (22). A "socket former" for the selected implant size was used to prepare the implant site. A "green-stick fracture" of the sinus floor was accomplished by hand tapping the "socket former" in a vertical direction. After preparation of the implant site, a root- formed implant was placed and allowed to heal in a submerged manner.

Summers (20) later described a different trans-alveolar approach, using set of tapered osteotomes with increasing diameters (Fig. 1). The concept intended to increase the density of soft, Type III and IV maxillary bone, resulting in better primary stability of inserted dental implants. Bone was conserved by this osteotome technique because drilling was not performed. Adjacent bone was compressed by pushing and tapping as the sinus membrane was elevated. Then, autogenous, allogenic or xenogenic grafts were added to increase the volume below the elevated sinus membrane.

Today, two main techniques for sinus floor elevation for dental implant placement are in use: a two-stage technique with a lateral window approach, followed by implant placement after a healing period, and a one-stage technique using either a lateral or trans-alveolar approach. The trans-alveolar approach, also referred to as "Osteotome Sinus Floor Elevation (OSFE)", the "Summers technique" or "Crestal approach" may be considered more conservative and less invasive than the conventional lateral approach of sinus floor elevation. A small osteotomy is performed through the alveolar crest of the edentulous ridge at the inferior border of the maxillary sinus. This intrusion osteotomy elevates the sinus membrane, thus creating a "tent". This provides space for graft placement and/or blood clot formation. It should be noted that the grafts are placed blindly into the space below the sinus membrane. Hence, the main disadvantage of this technique is the uncertainty of possible perforations of the sinus floor (Schneiderian) membrane. 


\section{Treatment options in the posterior maxilla}

Implant placement in the posterior maxilla remains a challenge. Reduced bone volume due to alveolar bone resorption and pneumatization of the sinus cavity makes it more difficult to place implants to support a dental prosthesis.

Several treatment options have been used in the posterior maxilla to overcome the problem of inadequate bone quantity. The most conservative treatment option would be to place short implants to avoid entering the sinus cavity. However, for the placement of short implants, there is still a need for at least $6 \mathrm{~mm}$ of residual bone height. Another way of avoiding grafting the maxillary sinus would be to place tilted implants mesially or distally to the sinus cavity if these areas have adequate bone. Furthermore, extra-long zygomatic implants may be placed in the lateral part of the zygomatic bone.

However, in patients with appropriate residual bone height, augmentation of the sinus floor can be accomplished via the trans-alveolar approach using the osteotome technique $(6,17,20)$. The problem of inadequate bone height can be overcome by elevating the maxillary sinus floor via the closed technique to provide sufficient quantity of bone for dental implant placement.

The most invasive treatment option in the posterior maxilla is the one- or two-stage sinus floor elevation with a lateral approach. By mastering these different methods, most edentulous areas in the maxilla can by restored with implant-supported reconstructions. The concept of a shortened dental arch must also be kept in mind. The work of Kayser (9) has shown that patients maintained adequate $(50-80 \%)$ chewing capacity with a premolar occlusion.

\section{Anatomy of the maxillary sinus}

The maxilla consists of various anatomic structures, including the maxillary sinus, the lateral nasal walls, the pterygoid plates, associated vasculature structures, and teeth.

The maxillary sinus maintains it's overall size while the posterior teeth remain in function. It is, however, well known, that the sinus expands with age, and especially when posterior teeth are lost. One or more septa termed "Underwood's septa" may divide the maxillary sinus into several recesses.

The overall prevalence of one or more sinus septa is between $26.5 \%$ and $31 \%(10,24)$ and is most common in the area between the second premolar and first molar. Edentulous segments have a higher prevalence of sinus septa than dentate maxillary segments.

The sinus is lined with respiratory epithelium (pseudo-stratified ciliated columnar epithelium) that covers a loose, highly vascular connective tissue. Underneath the connective tissue, immediately next to the bony walls of the sinus, is the periosteum. These structures (epithelium, connective tissue, and periosteum) are collectively referred to as the Schneiderian membrane. 


\section{Indications and contraindications}

The main indication for maxillary sinus floor elevation utilizing a trans-alveolar approach is reduced residual bone height, which does not allow standard implant placement.

Contraindications for trans-alveolar sinus floor elevation may by divided into three groups: intraoral contraindications, medical conditions, and local contraindications.

The medical contraindications include: chemotherapy or radiotherapy of the head and neck area at the time of trans-alveolar sinus floor elevation or in the preceding 6 months depending on the field of radiation; immunocompromised patients; medical conditions affecting bone metabolism; uncontrolled diabetes; drug or alcohol abuse; patient non-compliance; and psychiatric conditions. Whether or not smoking is an absolute contraindication for trans-alveolar sinus floor elevation remains controversial. A recent systematic review (16) investigated the influence of smoking on survival rate of implants inserted in combination with sinus floor elevation utilizing the lateral approach. Five of the included studies investigated the influence of smoking, although not clearly defined, on implant survival after sinus floor elevation. The patients were divided according to their smoking status. A group of non-smokers with 2159 implants and a group of smokers who received 863 implants were analysed. The group of smokers had a higher annual failure rate $(3.54 \%)$ compared with an annual failure rate of $1.86 \%$ for the non-smokers. However, this difference did not reach statistical significance in Possion regression analysis. In addition, patients with a history of inner ear complications and positional vertigo are not suitable for the osteotome technique.

Alteration of the nasal-maxillary complex that interferes with normal ventilation as well as the mucociliary clearance of the maxillary sinus, may be a contraindication for trans-alveolar sinus floor elevation. However, such abnormal conditions may be clinically asymptomatic or only present with mild clinical symptoms. These conditions include viral, bacterial, and mycotic rhinosinusitis, allergic sinusitis, sinusitis caused by intra-sinus foreign bodies, and odontogenic sinusitis resulting from necrotic pulp tissue. All odontogenic, periapical, and radicular cysts of the maxillary sinus should be treated prior to sinus floor elevation. A trans-alveolar sinus floor elevation under any of the above conditions may disturb the fine mucociliary balance, resulting in mucus stasis, suprainfection or a subacute sinusitis.

Local contraindications are inadequate residual bone height $(<4-5 \mathrm{~mm})$ and crestal bone width not allowing for sufficient primary stability of the implant. In addition, oblique sinus floor $\left(>45^{\circ}\right.$ inclination) is not suitable for the osteotome technique either (Fig. 2). The reason is the fact that the osteotomes first enter the sinus cavity at the lower level of an oblique sinus floor, while still having bone resistance on the higher level. In this situation, there is a high risk of perforating the sinus membrane with the sharp margin of the osteotome.

Absolute local contraindications for sinus floor elevation are: acute sinusitis; allergic rhinitis and chronic recurrent sinusitis; scarred and hypofunctional mucosae; local aggressive benign tumors; 
and malignant tumors.

\section{Surgical techniques}

Apart from the original Summers technique, only minor modifications have been presented $(4,7$, $15,17)$. The technique described in this article represents a modification of the original technique:

- Pre-surgical patient preparation includes oral rinsing with $0.1 \%$ chlorhexidine for a period of 1 minute.

- Local anesthesia is administered into the buccal and palatal regions of the surgical area.

- A mid-crestal incision with or without releasing incision is made and a full-thickness mucoperiosteal flap is raised.

- With a surgical stent or a distance indicator, the implant positions are marked on the alveolar crest with a small round bur (\#1). After locating the implant positions exactly, the opening of the preparations are widened with two sizes of round burs (\#2 and \#3) to a diameter about half a millimeter smaller than the implant diameter intended (Fig. 3).

- The distance from the crestal floor of the ridge to the floor of the maxillary sinus, measured prior to implant site preparation on the pre-operative radiograph may, in most cases, be confirmed at the time of surgery by penetrating the opening of the preparation with a blunt periodontal probe through the soft trabecular bone (type III or IV bone) to the floor of the maxillary sinus.

- After confirming the distance to the sinus floor, pilot drills with small diameters $(1-1.5 \mathrm{~mm}$ smaller than the intended implant diameter) are used to prepare the implant site to a distance of approximately $2 \mathrm{~mm}$ below from the sinus floor (Fig. 4). In conditions of soft type IV bone and a residual bone height of $5-6 \mathrm{~mm}$, there is usually no necessity to use the pilot drills. It is sufficient to perforate the cortical bone at the alveolar crest with the round burs.

- The first osteotome used in the implant site is a small diameter tapered osteotome with a rounded tip (Fig. 5). With light malleting, the osteotome is pushed towards the compact bone of the sinus floor (Fig. 6). After reaching the sinus floor, the osteotome is pushed about $1 \mathrm{~mm}$ further with light malleting in order to create a "greenstick" fracture on the compact bone of the sinus floor. A tapered osteotome with small diameter is chosen to minimize the force needed to fracture the compact bone.

- The second tapered osteotome also with a rounded tip, with a diameter slightly larger than the first one, is used to increase the fracture area of the sinus floor (Fig. 7). The second osteotome is applied to the same length as the first one.

- The third osteotome used is a straight osteotome with a diameter of about 1-1.5 mm smaller than the implant to be placed (Fig. 8). Instead of using the osteotomes to fracture the sinus floor, it has also been propagated to use piezoelectric surgery (Fig. 9). The advantage of this 
technique is that the perforation of the sinus floor may be achieved in a more controlled way than with osteotomes, and the risk of membrane perforation is reduced (ref). Moreover, this could reduce the risk of benign paroxysmal positional vertigo. The main disadvantage of this technique is that it is more time consuming than malleting, especially when the cortical bone at the sinus floor is relatively thick.

From this point onwards, the technique utilized in the surgical procedure depends on whether or not grafts will be placed.

\section{Implant placement without grafting material}

- Without applying grafting material, the straight osteotome, with a diameter about 1-1.5 mm smaller than that of the implant, will be pushed further until it penetrates the sinus floor.

- The last osteotome to be used should have a form and diameter suitable for the implant to be placed. For example, for a cylindrical implant with a diameter of $4.1 \mathrm{~mm}$, the last osteotome should be a straight osteotome with a diameter about $0.5 \mathrm{~mm}$ smaller than the implant diameter. It is important that the last osteotome only enters the preparation site once. If several attempts have to be made in sites with soft bone (type III or IV), there is a risk of increasing the diameter of the preparation which may jeopardize good primary stability. On the other hand, if the last osteotome diameter is too small compared to the implant diameter, too much force must be used to insert the implant. By squeezing the bone, more bone trauma and hence, greater bone resorption will occur delaying the osseointegration process (1). It is thus important, especially when placing implants in sites with reduced bone volume, that a fine balance between good primary stability and traumatizing the bone is respected.

- During the entire preparation, it is crucial to maintain precise control of the penetration length. Regular osteotomes have sharp cutting edges, thus entry into the sinus cavity increases the risk of membrane perforation. The final step before placing the implant is to check that the preparation is patent to the planned insertion depth. An osteotome with a rounded tip or a depth gauge with a relevant diameter is pushed to the decided length (Fig. 10).

\section{Implant placement with grafting materials}

- When performing the osteotome technique with grafting materials, the osteotomes are not supposed to enter the sinus cavity per se. Repositioned bone particles, grafting materials, and the trapped fluid will create a hydraulic effect moving the fractured sinus floor and the sinus membrane upwards. The sinus membrane is less likely to tear under this kind of pressure that has a fluid consistency.

- After pushing the third osteotome up to the sinus floor and before placing of any grafting material, the sinus membrane must by tested for any perforations. This is tested with the 
Valsalva maneuver (nose blowing). The nostrils of the patients are compressed (Fig. 11), and the patient blows the nose against the resistance. If air leaks out of the implant site, the sinus membrane is perforated, and no grafting material should be placed into the sinus cavity.

- If no air leaks out, the sinus membrane is intact and the preparation is filled with grafting material (Fig. 12). The grafting material is slowly pushed into the sinus cavity with the same straight third osteotome (Fig. 13). This procedure is repeated four to five times until about 0.2$0.3 \mathrm{~g}$ of grafting material has been pushed into the sinus cavity below the sinus membrane (Fig. 14). In the fourth and fifth time of applying grafting material, the tip of the osteotome may enter about $1 \mathrm{~mm}$ into the maxillary sinus cavity to test if there is resistance in the preparation site.

- Finally, before implant placement (Fig. 15), the preparation is checked for patency, as mentioned before, and the Valsalva maneuver is repeated.

To achieve good primary stability in the soft trabecular bone on the posterior maxilla implants with slight tapered configuration or implants with a tulip shaped neck are recommended. However, it must always be kept in mind, that applying too much force on the bone will result in greater bone resorption, delaying the osseointegration process (1).

\section{Post-surgical care}

The post-surgical care after the implant placement with the osteotome technique is similar to post-surgical care after standard implant placement. To minimize the post-operative discomfort, the surgical intervention should be carried out as atraumatically as possible. Precautions must be taken to avoid perforation of the flap and the sinus membrane. The bone should be kept moist during the surgery, and a tension-free primary flap closure is essential.

In addition to the standard oral home care, antiseptic rinsing with $0.1-0.2 \%$ chlorhexidine twice daily for the first three weeks after surgery is recommended. Although there are no studies available comparing post-surgical care with and without the use of prophylactic antiobiotics, it has been recommended in cases where bone substitutes were used. The patients were advised to take antibiotic prophylaxis e.g. Clamoxyl® $750 \mathrm{mg}$ three times daily, for a period of one week. Furthermore, antiseptic rinses with $0.1-0.2 \%$ chlorhexidine twice daily are indicated for the first 3 weeks after surgery.

\section{Complications}

When performing trans-alveolar sinus floor elevation, the risk of complications must be considered and the appropriate treatment foreseen. During trans-alveolar sinus floor elevation the intrusion osteotomy procedure elevates the sinus membrane, thus creating a "tent". This creates space for the blood clot and/or grafting material. An endoscopic study has shown that the sinus floor can be elevated up to $5 \mathrm{~mm}$ without perforating the membrane (5). It should be noted that the 
bone grafts are placed blindly into the space below the sinus membrane. Hence, the main disadvantage of this technique is the uncertainty of possible perforation of the sinus membrane. This constitutes the most common intra-operative complication. Presence of maxillary sinus septa and root apices penetrating into the sinus may increase the risk of membrane perforation. In a recent systematic review, addressing the trans-alveolar sinus floor elevation (21), 8 studies with 1621 implants out of the 19 included studies presented data on the incidence of perforation of the Schneiderian membrane. It varied between $0 \%-21.4 \%$, with a mean of $3.8 \%$.

Smaller perforations may be closed through the trans-alveolar preparation by using tissue fibrin glue. In cases of larger perforations, an access must be accomplished through a lateral window and barrier membranes, lamellar bone plates or suturing used either alone or in combination with tissue fibrin glue to close the membrane perforation. If the perforation occurs before any grafting material is inserted, the procedure should be aborted and a second attempt for a trans-alveolar sinus floor elevation may be performed 6-9 months later $(23,25)$.

Post-operative infection after trans-alveolar sinus floor elevation is a rare complication. Six studies, with 884 implants included in the systematic review of Tan and coworkers (2008), reported on post-operative infection. The incidence ranged between $0 \%$ and $2.5 \%$ with a mean of $0.8 \%$. Other complications reported were post-operative hemorrhage, nasal bleeding, blocked nose, hematomas, and loosening of cover screws resulting in suppuration and benign paroxysmal positional vertigo (BPPV). The BPPV may cause a substantial stress for the patient if not correctly identified and properly managed (26). No air embolism was reported in the study using hydraulic sinus condensing (4).

\section{Grafting materials}

In the original publication (22), the author did not use any grafting material to increase and maintain the volume of the elevated area.

Later on, Summer (20) described the Bone-Added Osteotome Sinus Floor Elevation (BAOSFE) technique, frequently referred to as the "Summers technique". Tapered osteotomes with increasing diameters were used to compress the bone and pushing and tapping it in a vertical direction as the sinus membrane was elevated. Autogenous, allogenic or xenogenic grafting material was added to maintain the volume below the elevated sinus membrane.

Grafting material is added incrementally to the osteotomy site and condensed until the desired graft height is reached. Pressure from the osteotomes is caused by the graft material and trapped fluids to exert hydraulic pressure on the sinus membrane, resulting in an elevation over a greater area (4). A recent study (11) comparing the use of Bone-Added Osteotome Sinus Floor Elevation with sinus floor elevation utilizing the lateral approach concluded minimal bone resorption seen for both methods. The reported bone resorption was $1.35 \mathrm{~mm}$ for the BAOSFE and $1.36 \mathrm{~mm}$ for 
the lateral approach over a period of two years after the procedure was performed.

From the 19 studies included in the systematic review mentioned (21), 15 of them used grafting material. Deproteinized bovine bone mineral (DBBM) was used in 5 studies, autogenous bone graft was used in 2 studies and collagen was used in another 2 studies. Five studies used combinations of grafts, with one study using autogenous bone graft and Bioglass $®$, one study used a combination of autogenous bone graft and DBBM, one study using autogenous bone graft and collagen, one study using autogenous bone graft, deminerialized freeze-dried bone allograft and tri-calcium phosphate, and another study using autogenous bone graft, deminerialized freeze-dried bone allograft and antibiotics in the graft. Various types of grafts were used in 2 studies. Three studies performed the procedure without graft placement and one study did not report on the graft used.

It is still controversial, whether or not it is necessary to apply grafting material to maintain the space for new bone formation after elevating the sinus membrane utilizing the trans-alveolar osteotome technique.

Studies in monkeys (2) showed that implants protruding into the maxillary sinus following elevation of the sinus membrane without grafting material, exhibited spontaneous bone formation over more than half of the implant's height. Hence, protrusion of an implant into the maxillary sinus does not appear to be an indication for bone grafting. In the same study, it was also seen that the design of the implant influenced the amount of spontaneous bone formation. Implants with open apices or deep-threaded configurations did not reveal substantial amounts of new bone formation. On the other hand, implants with rounded apices tended to show spontaneous bone formation extending all around the implants if they only penetrated 2-3 $\mathrm{mm}$ into the maxillary sinus. However, when the same implants penetrated $5 \mathrm{~mm}$ into the maxillary sinus, only a partial $(50 \%)$ growth of new bone was seen towards the apex of the implant.

A recent clinical study (13) reported similar clinical results. The authors reported on twenty-five $10 \mathrm{~mm}$ dental implants inserted using the trans-alveolar approach without grafting material. The implants protruded on average $4.9 \pm 1.9 \mathrm{~mm}$ into the sinus cavity after surgery. After a follow-up time of 5 years the implant protrusion was reduced to $1.5 \pm 0.9 \mathrm{~mm}$. Hence $3.4 \mathrm{~mm}$ or $70 \%$ of the penetrating part of the implants showed spontaneous bone formation.

In a clinical study (12), implants were installed into the sinuses of 40 patients using the transalveolar technique with no graft or cushion material. The authors reported a mean gain of alveolar bone height in scanned panoramic radiographs of $3.9 \pm 1.9 \mathrm{~mm}$.

In a retrospective study, assessing radiographically sinus floor remodeling after implant insertion using a modified trans-alveolar technique without grafting material (18), twenty-four patients were available for follow-up. Implant survival rate was $100 \%$. Bone filling around the implants was measured and compared with baseline digital radiographs. The mean height of the newly formed bone was $2.2 \pm 1.7 \mathrm{~mm}$ mesially and $2.5 \pm 1.5 \mathrm{~mm}$ distally, or, $86.3 \pm 22.1 \%$ and $89.7 \pm 13.3 \%$ of 
new bone formation, respectively.

In a prospective study (14), 252 implants were inserted using the trans-alveolar sinus floor elevation technique with or without grafting material. For $35 \%$ of these implants, deproteinized bovine bone mineral of particle size of $0.25-1 \mathrm{~mm}$ was used as grafting material, but for the remaining 164 implants, no grafting material was utilized. Periapical radiographs were made with a paralleling technique and digitized. Two investigators, that were blinded to whether grafting material was used or not, subsequently evaluated the pattern of tissue remodeling. The mean radiographic bone gain using the trans-alveolar technique with grafting material was significantly more or $4.1 \mathrm{~mm}$ (SD $2.4 \mathrm{~mm}$ ) compared with a mean bone gain of $1.7 \mathrm{~mm}$ (SD $2.0 \mathrm{~mm}$ ) when no grafting material was used (Fig. 16a, b).

\section{Success and implant survival}

A recent systematic review (21) analyzed the survival and complication rates of implants inserted in combination with trans-alveolar sinus floor elevation. An electronic search was conducted to identify prospective and retrospective cohort studies on trans-alveolar sinus floor elevation, with a mean follow-up time of at least 1 year after functional loading. The search provided 849 titles. Full-text analysis was performed for 176 articles resulting in 19 studies meeting the inclusion criteria. Meta-analysis of these studies indicated an estimated annual failure rate of $2.48 \%$ (95 percent confidence interval (95\% C.I.: $1.37 \%-4.49 \%)$ translating to an estimated survival rate of 92.8\% (95\% C.I.: $87.4 \%-96.0 \%)$ for implants placed in trans-alveolarly augmented sinuses, after 3 years in function (Table 1). Furthermore, subject-based analysis revealed an estimated annual failure of $3.71 \%$ (95\% C.I.: $1.21 \%-11.38 \%$ ), translating to $10.5 \%$ (95\% C.I.: $3.6 \%-28.9 \%$ ) of the subjects experiencing implant loss over 3 years.

\section{Residual bone height}

Of the 900 patient records that were screened for the Consensus Conference in 1996, only 100 had radiographs of adequate quality for analysis of the residual bone height. In total, only 145 sinus grafts in 100 patients, with 349 implants, were analyzed regarding the residual bone height. After a mean follow-up period of 3.2 years, 20 implants were lost. Of the implants lost, 13 were initially placed in residual bone with a height $4 \mathrm{~mm}$, seven were placed in residual bone with a height of $5-8 \mathrm{~mm}$. None of the implants placed in residual bone height of more than $8 \mathrm{~mm}$ was lost. There was a statistically significant difference in implant loss when residual bone height was $4 \mathrm{~mm}$ or less as compared to $5 \mathrm{~mm}$ or greater (8). Hence, for implants placed in combination with sinus floor elevation using the lateral approach to add to the residual bone height plays a significant role in implant survival. 
It is also evident that failure rate of the implants placed into sites with trans-alveolar sinus floor augmentation increases and correlates with reduced residual bone height and reduced implant length, as described in a multicenter retrospective study (17) that reported a survival rate of $96 \%$, when residual bone height was $5 \mathrm{~mm}$ or more. It decreased to $85.7 \%$, when residual bone height was $4 \mathrm{~mm}$ or less. Similar results were also reported in a recent prospective study (15) were $20 \%$ of the implants in the present study were placed in sites with residual bone height of $\leqslant$ $5 \mathrm{~mm}$. This, in turn, means that the limit of the osteotome technique has indeed been tested. The survival rates were $91.3 \%$ for implant sites with $\leqslant 4 \mathrm{~mm}$ residual bone height and $90 \%$ for sites with residual bone height between 4 and $5 \mathrm{~mm}$, compared with a survival rate of $100 \%$ if the residual bone height was above $5 \mathrm{~mm}$. Moreover, for short $6 \mathrm{~mm}$ implants, the survival rate was only $48 \%$. This clearly demonstrated that the trans-alveolar sinus floor elevation technique was most predictable with residual alveolar bone height of $5 \mathrm{~mm}$ or more and with implants of $8 \mathrm{~mm}$ or more.

\section{Patient-centered outcomes}

In the study of Pjetursson and coworkers (15), 163 patients were examined at the follow-up visit and asked to express their opinion about 9 statements related to the treatment. The first two statements dealt with general satisfaction with the treatment. The patients were asked if they would undergo similar treatment again if needed; On the Visual Analog Scale (VAS), the mean VAS score was $91 \pm 17$. The median was 98 (range $0-100$ ). The patients were also asked if they would recommend this treatment to a friend or relative, if indicated. On the VAS, the mean VAS score was $90 \pm 17$. The median was 97 (range $0-100$ ). For both statements only 5 or $3 \%$ of the patients would not be willing to undergo such a treatment again, respectively.

Approximately $23 \%$ of the patients found the surgical experience unpleasant. When asked about other surgical complications, $5 \%$ of the patients felt their head was tilted too far back during the surgery, $5 \%$ of the patients experienced vertigo, nausea and felt disoriented after the surgical procedure, but no patient had any problem with unusual eye movements. A small group of 5 patients had psychological problems after the treatment and had to seek medical assistance (15). The authors concluded that even though $23 \%$ of the patients reported the surgical procedure as unpleasant, more than $90 \%$ of the patients were be willing to undergo implant therapy again if necessary and dentally indicated.

\section{Research recommendations}

The present systematic review revealed several shortcomings in the clinical studies. Many of the studies on the survival of implants placed in sinus grafted sites failed to report the original residual bone height and graft failures, if any. 
There is a need for long-term clinical trial ( $\geqslant 5$ years) of implants inserted in combination with trans-alveolar sinus floor elevation. However, the question, whether different surgical techniques, implant types, and grafting materials would improve the outcomes, remains open and future research is needed. Randomized controlled clinical trials with sufficient statistical power comparing trans-alveolar sinus floor elevations with and without grafting materials would be of great value.

\section{Conclusions and clinical suggestions}

In the posterior maxilla, implants with morphometry designed to achieve high initial stability and with rough surface geometry giving high percentage of bone-to-implant contact during initial healing phase (1), should be preferred. Implants with slightly conical morphometry or implants with wider implant neck tend to give better primary stability in case of reduced residual bone height and soft bone geometry.

The clinical decision on which method (short implants, osteotome technique or lateral approach) should be chosen, depends on the residual bone height of the alveolar crest and on the surgeons' preferences. The following recommendations are suggested:

1. Residual bone height of $8 \mathrm{~mm}$ or more and flat sinus floor: standard implant placement installing relatively short implants.

2. Residual bone height of $8 \mathrm{~mm}$ or more and oblique sinus floor: standard implant placement using short implant or elevation of the maxillary sinus floor using the osteotome technique without grafting material.

3. Residual bone height of $5-7 \mathrm{~mm}$ and flat sinus floor: elevation of the maxillary sinus floor using the osteotome technique with grafting material that is resistant to resorption (Fig. 17).

4. Residual bone height of 5-7 mm and oblique sinus floor: elevation of the maxillary sinus floor using lateral approach with grafting material, and simultaneous implant placement (one-stage)

5. Residual bone height of 3-4 mm and flat or oblique sinus floor: elevation of the maxillary sinus floor using lateral approach with grafting material, and simultaneous implant placement (onestage).

6. Residual bone height of $1-2 \mathrm{~mm}$ and flat or oblique sinus floor: elevation of the maxillary sinus floor using lateral approach with grafting material and delayed implant placement 4-8 months later (two-stage). 


\section{References}

1. Abrahamsson I, Berglundh T, Linder E, Lang NP, Lindhe J. Early bone formation adjacent to rough and turned endosseous implant surfaces. An experimental study in the dog. Clin Oral Implants Res 2004: 15: 381-392

2. Boyne PJ. Analysis of performance of root-form endosseous implants placed in the maxillary sinus. J Long-Term Eff of Med Implants 1993: 3: 143-159

3. Boyne PJ, James R. Grafting of the maxillary sinus floor with autogenous marrow and bone. J Oral Surg 1980: 38: 613-618

4. Chen L, Cha J. An 8-year retrospective study: 1,100 patients receiving 1,557 implants using the minimally invasive hydraulic sinus condensing technique. J Perio 2005: 76: 482-491

5. Engelke W, Deckwer I. Endoscopically controlled sinus floor augmentation. A preliminary report. Clin Oral Implants Res 1997: 8: 527-531

6. Ferrigno N, Laureti M, Fanali S. Dental implant placement in conjunction with osteotome sinus floor elevation: 12-year life-table analysis from a prospective study on 588 ITI implants. Clin Oral Implants Res 2006: 17: 194-205

7. Fugazzotto PA. The modified trephine/osteotome sinus augmentation technique: technical considerations and discussion of indications. Implant Dent 2001: 10: 259-264

8. Jensen OT, Shulman LB, Block MS, lavono VJ. Report of the Sinus Consensus Conference of 1996. Int J Oral Maxillofac Implants 1996: 13(Suppl): 11-45

9. Kayser AF. Shortened dental arches and oral function. J Oral Rehabil 1981: 8: 457-462

10.Kim MJ, Jung UW, Kim CS, Kim KD, Choi SH, Kim CK, Cho KS. Maxillary sinus septa: prevalence, height, location, and morphology. A reformatted computed tomography scan analysis. J Perio 2006: 77: 903-908

11. Kim SM, Park JW, Suh JY, Sohn DS, Lee JM. Bone-added osteotome technique versus lateral approach for sinus floor elevation: a comparative radiographic study. Implant Dent 2011:

20: $465-470$

12. Leblebicioglu B, Ersanli S, Karabuda C, Tosun T, Gokdeniz H. Radiographic evaluation of dental implants placed using an osteotome technique. J Perio 2005: 76: 385-390

13. Nedir R, Nurdin N, Vazquez L, Szmukler-Moncler S, Bischof M, Bernard JP. Osteotome sinus floor elevation technique without grafting: a 5-year prospective study. J Clin Periodontol 2010:

37: 1023-1028

14. Pjetursson BE, Ignjatovic D, Matuliene G, Brägger U, Schmidlin K, Lang NP. Maxillary sinus floor elevation using the osteome technique with or without grafting material. Part II Radiographic tissue remodeling. Clin Oral Implants Res 2009: 20: 677-683

15. Pjetursson BE, Rast C, Brägger U, Zwahlen M, Lang NP. Maxillary sinus floor elevation using the osteome technique with or without grafting material. Part I - Implant survival and patient's perception. Clin Oral Implants Res 2009: 20: 667-676 
16. Pjetursson BE, Tan WC, Zwahlen M, Lang NP. A systematic review of the success of sinus floor elevation and survival of implants inserted in combination with sinus floor elevation. J Clin Periodontol 2008: 35 Suppl: 216-40

17. Rosen PD, Summers R, Mellado JR, Salkin LM, Shanaman RH, Marks MH, Fugazzotto PA. The bone-added osteotome sinus floor elevation technique: multicenter retrospective report of consecutively treated patients. Int J Oral Maxillofac Implants 1999: 14: 853-858

18. Schmidlin PR, Müller J, Bindl A, Imfeld H. Sinus floor elevation using an osteotome technique without grafting materials or membranes. Int J Periodontics Restorative Dent 2008: 28: 401-409

19. Sohn DS, Lee JS, An KM, Choi BJ. Piezoelectric internal sinus elevation (PISE) technique: a new method for internal sinus elevation. Implant Dent 2009: 18: 458-463

20. Summers RB. A new concept in maxillary implant surgery: the osteotome technique. Comp Continuing Educ Dent 1994: 15: 152-162

21. Tan WC, Lang NP, Zwahlen M, Pjetursson BE. A systematic review of the success of sinus floor elevation and survival of implants inserted in combination with sinus floor elevation. Part II: transalveolar technique. J Clin Periodontol 2008: 35(Suppl): 241-54

22. Tatum H. Maxillary and sinus implant reconstructions. Dent Clin North Am 1986: 30: 207229

23. Tatum $\mathrm{OH}$, Lebowitz MS, Tatum CA, Borgner RA. Sinus augmentation: rationale, development, long-term results. N Y State Dent J 1993: 59: 43-48

24. Ulm CW, Solar P, Gsellmann B, Matejka M, Watzek G. The edentulous maxillary alveolar process in the region of the maxillary sinus - a study of physical dimension. J Oral Maxillofac Surg 1995: 24: 279-282

25. Van den Bergh JP, ten Bruggenkate CM, Disch FJ, Tuinzing DB. Anatomical aspects of sinus floor elevations. Clin Oral Implants Res 2000: 11: 256-265

26. Vernamonte S, Mauro V, Vernamonte S, Messina AM. An unusual complication of osteotome sinus floor elevation: benign paroxysmal positional vertigo. Int J Oral Maxillofac Surg 2011: 40: 216-218 


\section{Legends}

Fig. 1 - In 1994, Summers introduced a set of tapered osteotomes with different diameters to compress and push the residual bone from the implant preparation into the sinus cavity and to elevate the sinus membrane.

Fig. 2 - The oblique inferior border of the maxillary sinus lies at approximately $60^{\circ}$ to the inferior border of the alveolar crest (the dotted lines represent the outlines of the residual bone). In a clinical situation like this, it is difficult to elevate the maxillary sinus floor with osteotomes. The osteotomes will first enter the sinus cavity distally at the lowest level of oblique sinus floor while still having bone resistance on the cranial level of the sinus floor. Hence, the risk of the sharp margin perforating the sinus membrane is high.

Fig. 3 - The exact position of the implant site is first marked with a small round bur (\#1) and then extended with two sizes of round burs (\#2 and \#3) to a diameter about 0.5-1 mm smaller than that of the implant to be installed.

Fig. 4 - The implant site is prepared to a distance of approximately $2 \mathrm{~mm}$ below the sinus floor with a small diameter pilot drill.

Fig. 5 - The first osteotome used in the implant site is a small-diameter tapered osteotome. Such an osteotome is chosen to minimize the force needed to fracture the compact bone.

Fig. 6 - After reaching the sinus floor, the osteotome is pushed approximately $1 \mathrm{~mm}$ further with light malleting in order to create a "greenstick" fracture on the compact bone of the sinus floor.

Fig. 7 - A second osteotome, which is also tapered, but with a diameter slightly larger then the first one, is used to increase the fractured area of the sinus floor.

Fig. 8 - The last osteotome to be used must have a form and diameter suitable for the implant to be placed. For example, for a cylindrical implant with a diameter of $4.1 \mathrm{~mm}$, the last osteotome should be straight with a diameter approximately $0.5 \mathrm{~mm}$ smaller than that of the implant. It is important that the last osteotome is allowed to enter the preparation site only once.

Fig. 9 - A kit of diamante coated insertion tips for piezosurgery that can be used to prepare the implant site and to trim down or perforated the cortical bone at the lower border of the maxillary sinus. 
Fig. 10 - The final step before placing the implant is to check that the preparation is patent to the planned insertion depth. Osteotome with a rounded tip or a depth gauge, for the relevant implant diameter, is pushed to the decided length.

Fig. 11 - To test the sinus membrane for perforations, the nostrils of the patients are compressed and the patient is asked to blow his nose. If air leaks out of the implant site, the sinus membrane is perforated, and no grafting material should be placed into the sinus cavity.

Fig. 12 - If the sinus membrane is intact, the preparation site is filled four to five times with grafting material.

Fig. 13 - The grafting material is then slowly pushed into the sinus cavity with a straight osteotome with a diameter about $1-1.5 \mathrm{~mm}$ smaller than that of the intended implant size.

Fig. 14 - Grafting material is slowly pushed into the sinus cavity with a straight osteotome. The tip of the osteotome is only supposed to enter the sinus cavity after some grafting material has been pushed through the preparation site to elevate the sinus membrane.

Fig. 15 - A rough-textured implant was installed after preparing the implant site with the osteotome technique. To achieve good primary stability implants with slight tapered configuration or implants with a tulip shaped neck are recommended.

Fig. 16 - (a) A radiograph, taken at the 5-year follow-up visit, of an implant placed in the 1st quadrant utilizing the osteotome technique without grafting material A new cortical bony plate at the inferior border of the maxillary sinus is clearly visible, but no bony structure can be detected apical to the implant (b) A radiograph (same patient) of an implant placed in the 2nd quadrant utilizing the osteotome technique with xenograft grafting material, taken after 5 years in function A dome-shaped structure is clearly visible documenting a definite increase in bone volume compared to the initial situation The "dome" is surrounded with a new cortical bony plate

Fig. 17 - The ideal indication for the trans-alveolar sinus floor elevations are sites with residual bone height of $5-7 \mathrm{~mm}$ and relatively flat sinus floor anatomy. The radiograph, taken after implant placement, showing a dome-shape configuration of the graft. In this instance, $0.25 \mathrm{~g}$ of grafting material (xenograft) was used to elevate the sinus membrane (the dotted lines represent the outlines of the residual bone) 